\title{
The structure and robustness of nocturnal Lepidopteran pollen-transfer networks in a Biodiversity Hotspot
}

\author{
PAULA BANZA, ${ }^{1,2}$ ANABELA D. F. BELO ${ }^{3}$ and DARREN M. EVANS ${ }^{4}{ }^{1}$ ICAAM - Instituto \\ de Ciências Agrárias e Ambientais Mediterrânicas, IIFA - Instituto de Investigação e Formação Avançada, Universidade de \\ Évora, Évora, Portugal, ${ }^{2}$ A Rocha Portugal, Mexilhoeira Grande, Portugal, ${ }^{3}$ Departamento de Biologia, Escola de Ciências e \\ Tecnologia, ICAAM - Instituto de Ciências Agrárias e Ambientais Mediterrânicas, Universidade de Évora, Évora, Portugal \\ and ${ }^{4}$ School of Biological, Biomedical and Environmental Sciences, University of Hull, Hull, UK
}

\begin{abstract}
The role of nocturnal moths within plant-pollinator networks is poorly understood but could be important in the context of declining biodiversity and the ecosystem services they provide.

2. For the first time, this study examined the role of moths as pollen vectors in the Mediterranean Biodiversity Hotspot. Light traps were used to sample moths in SW Portugal in 2010. The pollen on moth head parts was collected, identified, and counted to construct a nocturnal pollen-transfer and flower-visitor network.

3. A total of 257 moths belonging to 95 species were captured in 11 trapping sessions in 2010; 196 moths $(76 \%)$ carried pollen and the total number of pollen grains counted and identified was 9064 .

4. The pollen-transfer network exhibited a high degree of selectivity $\left(\mathrm{H} 2^{\prime}\right)$ but low robustness when the most-to-least connected plants were made extinct in the network. The flower-visitor network (based on the incidences of interactions by individual moths), however, exhibited high linkage density and was generally more robust to simulated plant or moth extinction.

5. Including nocturnal moths in plant-pollinator networks will provide a better understanding of their robustness to species extinctions due to environmental change as well as the impacts on ecosystem structure and functioning. Nocturnal pollen-transfer networks could be developed for identifying key species for targeted conservation.
\end{abstract}

Key words. Ecological network analysis, food-webs, interactions, Mediterranean region, moths, plant-pollinator networks, pollen transport.

\section{Introduction}

Ecological networks describe the interactions between species, the underlying structure of communities and the function and stability of ecosystems (Montoya et al.,

Correspondence: Darren M. Evans, School of Biological, Biomedical and Environmental Sciences, University of Hull, Room 317 Hardy Building, Cottingham Road, Hull HU6 7RX, UK. Email: d.evans@hull.ac.uk
2006). In recent years, a burgeoning interest in insectflower interactions has provided valuable insights into the structuring of mutualistic networks (Memmott, 1999; Bascompte et al., 2003; Fontaine et al., 2006; Memmott et al., 2007), their robustness to species extinction (Memmott et al., 2004; Kaiser-Bunbury et al., 2010; Pocock et al., 2012) as well as the impacts of environmental change (Fortuna \& Bascompte, 2006; Lopezaraiza-Mikel et al., 2007; Memmott et al., 2010; Evans et al., 2013). With pollinating insects experiencing significant declines in many parts of the world (Biesmeijer et al., 2006; An \& 